\title{
Aggregation behavior of polystyrene-based amphiphilic diblock copolymers in organic media
}

\author{
Tomoe Arai, Makoto Masaoka, Tomohiro Michitaka, Yosuke Watanabe, Akihito Hashidzume \\ and Takahiro Sato
}

Two polystyrene (PS)-based amphiphilic diblock copolymers, $\mathrm{PS}(x)-\mathrm{PV}(y)$ with $x / y=3.3-7.7$ and $\mathrm{PS}(x)$-PA(y) with $x / y=1.2-13.7$ (where PS $(x)-\operatorname{PV}(y)$ and PS $(x)$-PA(y) possess poly[(ar-vinylbenzyl)trimethylammonium chloride] and poly(2acrylamido-2-methylpropanesulfonic acid) blocks, respectively, and $x$ and $y$ are the degrees of polymerization), were prepared by reversible addition-fragmentation chain transfer radical polymerization to investigate the formation of reverse micelles by these copolymers in less polar organic media. The $\mathrm{PS}(x)-\mathrm{PV}(y)$ samples were more dispersible in halogenated solvents, for example, chloroform and 1,2-dichloroethane (DCE), than the PS $(x)$-PA(y) samples because of the higher solvophobicity of the PA block. ${ }^{1} \mathrm{H}$ NMR spectra for the $\mathrm{PS}(x)-\mathrm{PV}(y)$ and $\mathrm{PS}(x)-\mathrm{PA}(y)$ samples measured in $\mathrm{CDCl}_{3}$ exhibited no signals that corresponded to the PV or PA blocks in the diblock copolymers, which indicated that the PS $(x)-\operatorname{PV}(y)$ and $\operatorname{PS}(x)-\operatorname{PA}(y)$ samples formed reverse micelles in halogenated solvents with the PV or PA blocks in the core. The structures of the reverse micelles formed from the PS $(x)-\operatorname{PV}(y)$ samples in DCE were investigated in detail by static and dynamic light scattering. The light scattering data indicated that PS $(x)$-PV $(y)$ formed star-like micelles at $x / y>6$ and non-star-like micelles at $x / y<6$ in DCE. The morphology of the micelles formed from PS $(x)-\operatorname{PV}(y)$ with $x / y<6$ in DCE was most likely brush-like.

Polymer Journal (2014) 46, 189-194; doi:10.1038/pj.2013.80; published online 25 September 2013

Keywords: aggregation behavior; polystyrene-based amphiphilic diblock copolymers; reversible addition-fragmentation chain transfer (RAFT) radical polymerization; reverse micelle

\section{INTRODUCTION}

Amphiphilic polymers possessing solvophilic and solvophobic units in the same polymer chain form various types of aggregates in selective solvents depending on the chemical structure of the polymer. $^{1-4}$ Their aggregation behavior is important not only because they are used in various applications including cosmetics, drug delivery systems, paints, coatings and personal care goods ${ }^{5-7}$ but also because they are useful as simple models for the formation of higher order structures of biological macromolecules. Therefore, the aggregation behavior of amphiphilic polymers has been studied by a number of research groups over the past two or more decades. ${ }^{1-4}$ According to the results of these studies, the aggregation behavior of amphiphilic polymers can be controlled by changing the chemical structure.

Amphiphilic diblock copolymers have been the most investigated because of their simple structures, similar to those of low-molecularweight surfactants. ${ }^{8-15}$ A larger number of studies on amphiphilic diblock copolymers have indicated that these copolymers form various types of aggregates (for example, star-like, brush-like and disk-like micelles and vesicles) in selective solvents. ${ }^{9-15}$ A large majority of recent studies on amphiphilic diblock copolymers have used aqueous solutions, which are of interest for environmentally benign systems. ${ }^{16-21}$ Recently, we have been focusing on reverse micelles formed by amphiphilic diblock copolymers in less polar organic media to utilize the hydrophilic core as a medium for chemical reactions. Because the type of hydrophilic unit may be critical for chemical reactions in the hydrophilic core, the formation of reverse micelles from amphiphilic diblock copolymers consisting of various hydrophilic blocks needs to be investigated.

Because styrene is a representative vinyl monomer, polystyrene (PS)-based amphiphilic diblock copolymers containing several hydrophilic blocks (for example, poly(ethylene oxide), poly(4-vinylpyridine), poly(2-vinylpyridine), poly(acrylic acid) and poly(methacrylic acid)) have been prepared, and the formation of reverse micelles by these copolymers has been investigated. ${ }^{22-30}$ These studies have elucidated the micellization behavior as a function of the ratio of the block lengths, but the effect of the type of hydrophilic block still needs to be clarified. In this study, we synthesized new PS-based amphiphilic diblock copolymers (that is, PS- $b$-poly[(ar-vinylbenzyl) trimethylammonium chloride] and PS- $b$-poly(2-acrylamide-2-methylpropanesulfonic acid), $\operatorname{PS}(x)-\operatorname{PV}(y)$ and $\operatorname{PS}(x)-\operatorname{PA}(y)$, respectively; Scheme 1) and investigated the formation of reverse micelles by these 


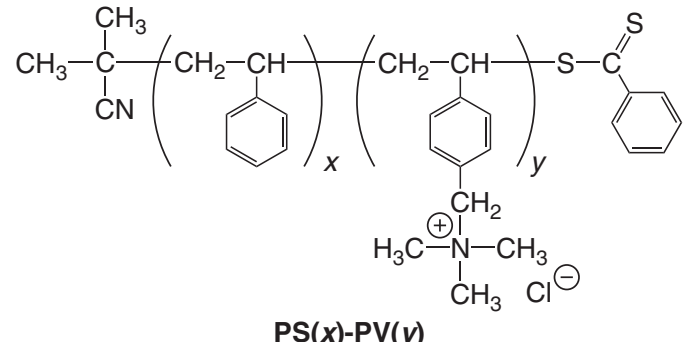

$\operatorname{PS}(x)-\operatorname{PV}(y)$

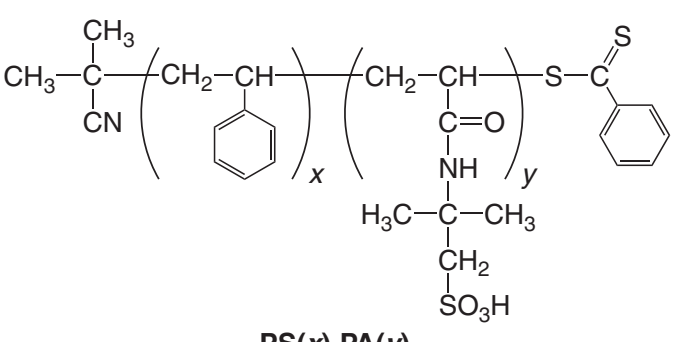

$\operatorname{PS}(x)-\operatorname{PA}(y)$

Scheme 1 Structure of the diblock copolymers used in this study, PS $(x)$ $\operatorname{PV}(y)$ and $\mathrm{PS}(x)-\mathrm{PA}(y)$

copolymers in 1,2-dichloroethane. The characterization data for the reverse micelles of $\operatorname{PS}(x)-\operatorname{PV}(y)$ were compared with the data for the reverse micelles of other PS-based amphiphilic diblock copolymers.

\section{EXPERIMENTAL PROCEDURE}

\section{Materials}

Styrene was purchased from Wako Pure Chemical Industries (Osaka, Japan) and purified by distillation under reduced pressure immediately before use. (ar-Vinylbenzyl)trimethylammonium chloride (VTAC) and 2-acrylamido-2methylpropanesulfonic acid (AMPS) were purchased from Sigma-Aldrich (St Louis, MO, USA) and Wako Pure Chemical Industries (Osaka, Japan), respectively, and used as received. 2,2'-Azobis(isobutyronitrile) (AIBN) was purchased from Kanto Chemical Co. (Tokyo, Japan) and purified by recrystallization from methanol. 1-Cyano-1-methylethyl dithiobenzoate (CMEDTB), which was used as a chain transfer agent (CTA), was prepared according to a previously reported procedure. ${ }^{31,32}$ Toluene and $\mathrm{N}, \mathrm{N}$ dimethylformamide (DMF), which were used for polymerization, were distilled over calcium hydride under reduced pressure immediately before use. 1,2-Dichloroethane (DCE), which was used for measurements, was purified by distillation. Other reagents were used without further purification.

\section{Preparation of diblock copolymer samples}

A typical example of the reversible addition-fragmentation chain transfer (RAFT) radical polymerization of styrene is described below. Styrene $(12.0 \mathrm{ml}$, $104 \mathrm{mmol})$, CMEDTB $(228 \mathrm{mg}, 1.03 \mathrm{mmol})$ and AIBN (34 mg, $0.21 \mathrm{mmol})$ were dissolved in toluene $(60 \mathrm{ml})$ using a $100-\mathrm{ml}$ flask equipped with a threeway stopcock under an argon atmosphere, and then the flask was warmed using an oil bath maintained at $80^{\circ} \mathrm{C}$ for $24 \mathrm{~h}$. The polymerization was terminated by rapid cooling with an ice-water bath. The reaction mixture was slowly poured into a large excess of methanol. The obtained polymer was recovered by filtration and dried under reduced pressure at room temperature (yield $2.5 \mathrm{~g}, 23 \%$ ).

A typical preparation for $\operatorname{PS}(x)-\operatorname{PV}(y)$ is described below. The obtained polystyrene (PS) (macro-CTA) $\left(M_{\mathrm{n}}=2.6 \times 10^{3}, 0.52 \mathrm{~g}, 0.20 \mathrm{mmol}\right)$, VTAC $(466 \mathrm{mg}, 2.2 \mathrm{mmol})$ and AIBN $(6.4 \mathrm{mg}, 0.039 \mathrm{mmol})$ were dissolved in DMF $(5.2 \mathrm{ml})$ in a test tube equipped with a three-way stopcock under an argon atmosphere, and then the tube was warmed with an oil bath maintained at $80^{\circ} \mathrm{C}$ for $24 \mathrm{~h}$. The polymerization was terminated by rapid cooling with an ice-water bath. The reaction mixture was slowly poured into a large excess of water to recover the prepared polymer. The polymer was dried under reduced pressure at room temperature (yield $0.67 \mathrm{~g}, 33 \%$ ).

\section{Measurements}

Size exclusion chromatography measurements of the PS samples were performed at $30^{\circ} \mathrm{C}$ using a system consisting of a Tosoh DP-8020 pump, a Viscotek TDA 302 detector and two Tosoh TSKgel GMH $\mathrm{XL}_{\mathrm{L}}$ columns connected in series using THF as the eluent at a flow rate of $1.0 \mathrm{ml} \mathrm{min}^{-1}$. The molecular weights were calibrated with polystyrene standards (Tosoh TSK standard POLYSTYRENE). The size exclusion chromatography measurements of the copolymers were performed at $35^{\circ} \mathrm{C}$ on a Shodex GPC-101 equipped with a Tosoh TSKgel GMH $\mathrm{XL}_{\mathrm{XL}}$ column using DMF as the eluent at a flow rate of $0.5 \mathrm{mlmin}^{-1}$. Shodex RI-71 and Wyatt DAWN HELEOS-II detectors were used. The sample solutions were filtered with a $0.20-\mu \mathrm{m}$ PTFE membrane filter immediately before performing the measurements.

${ }^{1} \mathrm{H}$ NMR spectra were collected on a JEOL JNM ECA500 or ECS400 spectrometer using dimethyl sulfoxide- $d_{6}\left(\mathrm{DMSO}-d_{6}\right)$ or $\mathrm{CDCl}_{3}$ as the solvent at $30^{\circ} \mathrm{C}$ or $50^{\circ} \mathrm{C}$.

Various solvents were added to the dried samples of PS $(x)-\operatorname{PV}(y)$ and $\operatorname{PS}(x)$ $\mathrm{PA}(y)$, and the mixtures were vigorously stirred at room temperature to examine the dispersibility of the samples in the solvents.

Simultaneous static and dynamic light scattering (SLS and DLS, respectively) experiments were performed for the copolymer solutions in DCE at $25.0^{\circ} \mathrm{C}$ using an ALV/SLS/DLS-5000 light scattering instrument equipped with an ALV-5000 multiple $\tau$ digital correlator using a Nd:YAG $(532 \mathrm{~nm})$ laser. The solutions of the $\operatorname{PS}(x)-\operatorname{PV}(y)$ and $\operatorname{PS}(x)-\operatorname{PA}(y)$ samples in DCE that were used in the light scattering measurements were optically cleaned by filtration through a $0.45-$ or $0.50-\mu \mathrm{m}$ PTFE membrane filter into a cell. The excess Rayleigh ratio $R_{\theta}$ obtained by SLS and the first cumulant $\Gamma$ obtained by DLS (with the CONTIN analysis ${ }^{32,33}$ ) for each solution were analyzed using the following standard equations: ${ }^{32-34}$

$$
\begin{aligned}
& \lim _{k \rightarrow 0}\left(K c / R_{\theta}\right)^{1 / 2}=M_{\mathrm{w}}^{-1 / 2}+A_{2} M_{\mathrm{w}}^{1 / 2} c+O\left(c^{2}\right) \\
& \lim _{c \rightarrow 0}\left(K c / R_{\theta}\right)^{1 / 2}=M_{\mathrm{w}}^{-1 / 2}\left[1+\frac{1}{6}\left\langle S^{2}\right\rangle_{\mathrm{z}} k^{2}+O\left(k^{4}\right)\right] \\
& \lim _{k, c \rightarrow 0} \Gamma / k^{2}=D=\frac{k_{\mathrm{B}} T}{6 \pi \eta_{0} R_{\mathrm{H}}}
\end{aligned}
$$

to determine the weight average molar mass $M_{\mathrm{w}}$, the second virial coefficient $A_{2}$, the z-average square radius of gyration $\left\langle S^{2}\right\rangle_{\mathrm{z}}$ and the hydrodynamic radius $R_{\mathrm{H}}$ of the aggregate in the solution. Here $K$ is the optical constant, $c$ is the copolymer mass concentration, $D$ is the diffusion coefficient, $k^{2}$ is the square of the scattering vector, $k_{\mathrm{B}} T$ is the Boltzmann constant multiplied by the absolute temperature and $\eta_{0}$ is the solvent viscosity coefficient.

The specific refractive index increment $\partial n / \partial c$ for each sample of PS $(x)$ $\operatorname{PV}(y)$ and $\operatorname{PS}(x)-\operatorname{PA}(y)$ in DCE was determined with a Shimadzu modified Schulz-Cantow type differential refractometer at $25.0^{\circ} \mathrm{C}$ to calculate the optical constant $K$ present in equations $1 \mathrm{a}$ and $\mathrm{b}$.

It is known that the true $M_{\mathrm{w}}$ is not obtained by light scattering when the copolymer sample has a composition distribution. This composition distribution effect is examined in the Supplementary Information. Because the correction factors for $M_{\mathrm{w}}$ were estimated to be near unity, we have neglected the composition distribution effect on $M_{\mathrm{w}}$ in the following discussion.

\section{RESULTS AND DISCUSSION}

In this study, amphiphilic block copolymers possessing a hydrophobic PS block were prepared by RAFT radical polymerization. Several PS samples with different degrees of polymerization $(x=23-69)$ and narrow molecular weight distributions $\left(M_{\mathrm{w}} / M_{\mathrm{n}}=1.07-1.16\right)$ were first prepared by RAFT radical polymerization using CMEDTB as a CTA in modest yield $(18-39 \%)$. Then, VTAC and AMPS were polymerized by RAFT radical polymerization using a prepared PS sample as a macro-CTA to yield $\operatorname{PS}(x)-\operatorname{PV}(y)$ and $\operatorname{PS}(x)-\operatorname{PA}(y)$ samples, respectively. 


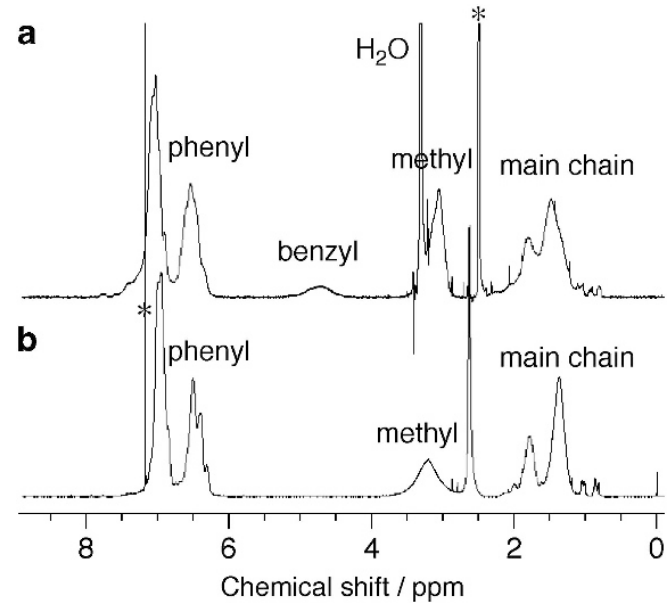

Figure $1{ }^{1} \mathrm{H}$ NMR spectra for PS(37)-PV(6) measured in DMSO-d $(a)$ and $\mathrm{CDCl}_{3}(\mathbf{b})$

Table 1 Molecular characteristics of the diblock copolymers used in this study

\begin{tabular}{lrrrrr}
\hline polymer code & $x^{\mathrm{a}}$ & $y^{\mathrm{a}}$ & $x / y^{\mathrm{a}}$ & $M_{n} / 10^{3}$ & $M_{w} / M_{n}^{\mathrm{b}}$ \\
\hline PS(23)-PV(7) & 23 & 7 & 3.3 & 4.1 & 1.70 \\
PS(55)-PV(10) & 55 & 10 & 5.5 & 8.1 & 1.75 \\
PS(69)-PV(12) & 69 & 12 & 5.8 & 10 & 2.06 \\
PS(37)-PV(6) & 37 & 6 & 6.2 & 5.3 & 1.97 \\
PS(30)-PV(4) & 30 & 4 & 7.5 & 4.2 & 1.74 \\
PS(23)-PV(3) & 23 & 3 & 7.7 & 3.3 & 1.53 \\
PS(41)-PA(38) & 41 & 38 & 1.2 & 13 & 1.18 \\
PS(41)-PA(7) & 41 & 7 & 5.9 & 6.1 & 1.15 \\
PS(43)-PA(7) & 43 & 7 & 6.1 & 6.3 & 1.37 \\
PS(41)-PA(4) & 41 & 4 & 10.3 & 5.4 & $-^{c}$ \\
PS(41)-PA(3) & 41 & 3 & 13.7 & 5.2 & C $^{c}$
\end{tabular}

Abbreviations: $M_{n}$, number average molecular weight; $M_{m}$ molecular weight

Determined by ${ }^{1} \mathrm{H}$ NMR in DMSO- $d_{6}$.

${ }^{\text {b}}$ Determined by size exclusion chromatography using DMF containing $0.05 \mathrm{M} \mathrm{LiClO}_{4}$ as eluent.

The molecular weights were calibrated with polystyrene standards.

cNot measured for a technical reason.

Figure 1a shows a typical example of the ${ }^{1} \mathrm{H}$ NMR spectra for PS(37)-PV(6) in DMSO- $d_{6}$, the common solvent. The spectrum contains signals that correspond to the benzyl and methyl protons in the VTAC units at ca. 4.7 and 3.0 p.p.m., respectively, to the phenyl protons in the PS units at ca. 6.5 p.p.m. and to the terminal AIBN fragment at ca. 1 p.p.m. Table 1 lists the degrees of polymerization $\left(x\right.$ and $y$ ) determined by ${ }^{1} \mathrm{H}$ NMR spectra for the $\operatorname{PS}(x)-\operatorname{PV}(y)$ and PS $(x)$-PA $(y)$ samples obtained and their number average molecular weights $M_{\mathrm{n}}$ calculated from $x$ and $y$ with the molar masses of PS $\left(M_{0, \mathrm{~S}}=104\right), \mathrm{PV}\left(M_{0, \mathrm{~V}}=211.5\right), \mathrm{PA} \quad\left(M_{0, \mathrm{~A}}=229\right)$, the initiator segment $\left(M_{\mathrm{I}}=68\right)$, and the CTA segment $\left(M_{\mathrm{T}}=153\right)$. Six samples of $\operatorname{PS}(x)-\operatorname{PV}(y)$ with $x / y=3.3-7.7$ and five samples of $\operatorname{PS}(x)-\operatorname{PA}(y)$ with $x / y=1.2-13.7$ were prepared and investigated. The ratios of the weight to number average molecular weight $M_{\mathrm{w}} / M_{\mathrm{n}}$ for all of the $\operatorname{PS}(x)-\operatorname{PV}(y)$ samples and three $\operatorname{PS}(x)-\operatorname{PA}(y)$ samples, which were determined by size exclusion chromatography using DMF containing $0.05 \mathrm{M} \mathrm{LiClO}_{4}$ as the eluent and calibrated with polystyrene standards, are also listed in Table 1.

Table 2 summarizes the results of the dispersibility test for the $\operatorname{PS}(x)-\operatorname{PV}(y)$ and $\operatorname{PS}(x)-\operatorname{PA}(y)$ samples in various solvents. All of the
Table 2 Results of the dispersibility test for the diblock copolymers used in this studya

\begin{tabular}{lccccccc} 
Polymer code & DMF & Toluene & Benzene & Ethyl acetate & Chloroform & DCE & Water \\
\hline PS(23)-PV(7) & D & ND & ND & ND & D & D & ND \\
PS(55)-PV(10) & D & ND & ND & ND & D & D & ND \\
PS(69)-PV(12) & D & ND & ND & ND & D & D & ND \\
PS(37)-PV(6) & D & ND & ND & ND & D & D & ND \\
PS(30)-PV(4) & D & ND & ND & ND & D & D & ND \\
PS(23)-PV(3) & D & ND & ND & ND & D & D & ND \\
PS(41)-PA(38) & D & ND & ND & ND & D & D & D \\
PS(41)-PA(7) & D & ND & ND & ND & D & D & ND \\
PS(43)-PA(7) & D & ND & ND & ND & D & D & ND \\
PS(41)-PA(4) & D & ND & ND & ND & D & D & ND \\
PS(41)-PA(3) & D & ND & ND & ND & D & D & ND
\end{tabular}

Abbreviations: DCE, 1,2-dichloroethane; DMF, $N, N$-dimethylformamide ${ }^{\mathrm{a} D}$ and ND denote dispersed and not dispersed, respectively.

PS $(x)-\mathrm{PV}(y)$ samples were dispersible in DMF and DMSO. However, the PS $(x)-\operatorname{PV}(y)$ samples were not dispersible in less polar solvents (that is, toluene, benzene and ethyl acetate) or in water. This characteristic is in contrast to the dispersibility of the nonionic block copolymer polystyrene- $b$-poly(4-vinylpyridine) (PS-P4VP) in toluene. ${ }^{11}$ $\operatorname{PS}(x)-\mathrm{PV}(y) \quad$ samples with $x / y=3.3-7.7$ were dispersible in halogenated solvents (that is, chloroform and DCE).

Figure $1 \mathrm{~b}$ shows a typical example of the ${ }^{1} \mathrm{H}$ NMR spectra obtained for PS(37)-PV(6) in $\mathrm{CDCl}_{3}$. The broad signal is because of the phenyl and methyl protons at ca. 6.5 and 3.2 p.p.m., respectively, and no signals corresponding to the benzyl protons were observed, which indicated that the mobility of the VTAC units was markedly restricted. Because the PS block is soluble and the PV block is insoluble in halogenated solvents, it is likely that the $\operatorname{PS}(x)-\operatorname{PV}(y)$ samples with $x / y=3.3-7.7$ form reverse micelles with the PV blocks in the core.

All of the PS $(x)-\operatorname{PA}(y)$ samples were also dispersible in the common solvent DMF. Because the PA and PS blocks are very hydrophilic and hydrophobic, respectively, all of the $\operatorname{PS}(x)-\operatorname{PA}(y)$ samples were not dispersible in toluene, benzene, ethyl acetate or water. However, except for PS(41)-PA(38), the $\operatorname{PS}(x)-\operatorname{PA}(y)$ samples were dispersible in chloroform and DCE even though the dispersions of the PS(41)$\mathrm{PA}(7)$ and PS(43)-PA(7) samples were very turbid. The dispersibility of the $\operatorname{PS}(x)-\operatorname{PA}(y)$ samples in halogenated solvents was much lower than that of $\operatorname{PS}(x)-\operatorname{PV}(y)$ because of the difference in the solvophobicity of the PV and PA blocks. The ${ }^{1} \mathrm{H}$ NMR spectra for the PS $(x)$ $\mathrm{PA}(y)$ samples with $x / y=10.3$ and 13.7 measured in $\mathrm{CDCl}_{3}$ exhibited very broad and weak signals because of the AMPS units (data not shown). On the basis of these observations, DCE was chosen as the organic solvent used in the investigation of the structure of the reverse micelles formed by $\operatorname{PS}(x)-\operatorname{PV}(y)$ and $\operatorname{PS}(x)-\operatorname{PA}(y)$ because it is easier to handle DCE than chloroform because of DCE's higher boiling point.

Figure 2 shows typical examples of the DLS and SLS results for PS(37)-PV(6) in DCE. The relaxation time spectrum $A(\tau)$ (Panel b) is unimodal, indicating that the solution contains only a single scattering component. In the plot of $\left(K c / R_{\theta}\right)^{1 / 2}$ as a function of $k^{2}$ (Panel a), the data points at each $c$ appear to be linear with a slightly negative slope, which may be due to the interparticle interference that has often been observed in micellar solutions of block copolymers. ${ }^{35,36}$ At infinite dilution (indicated by filled circles), the data points exhibit a nearly horizontal line. Figure 3 summarizes the concentration dependence of $\left(K c / R_{0}\right)^{1 / 2}\left(\left(K c / R_{\theta}\right)^{1 / 2}\right.$ extrapolated to 

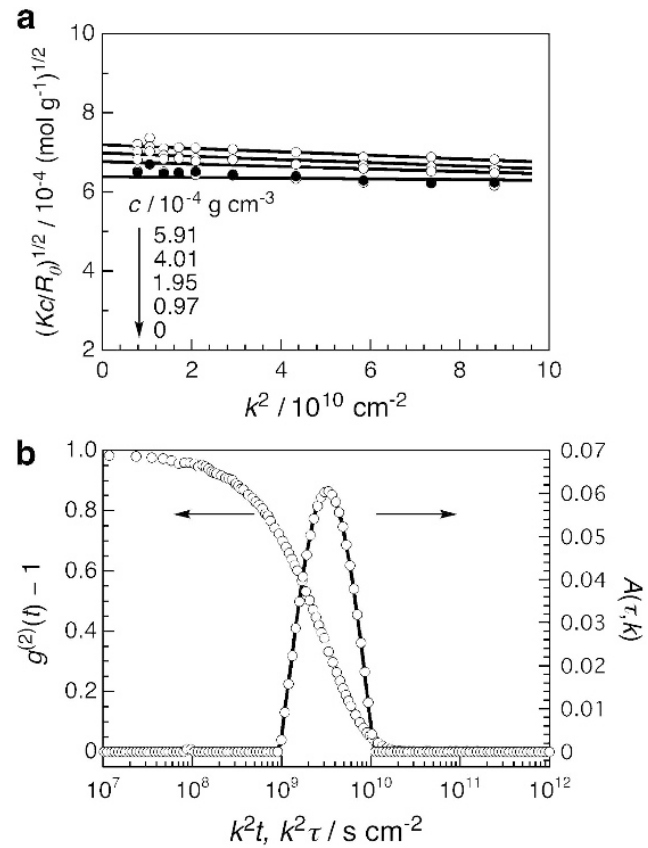

Figure 2 Angular dependences of $\left(K c / R_{\theta}\right)^{1 / 2}$ (a) and the autocorrelation function $g^{(2)}(t)$ and the relaxation time spectrum $A(t) \quad\left(\theta=90^{\circ}\right.$ and $c=0.006 \mathrm{~g} \mathrm{~cm}^{-3}$ ) for PS(37)-PV(6) in DCE (b).

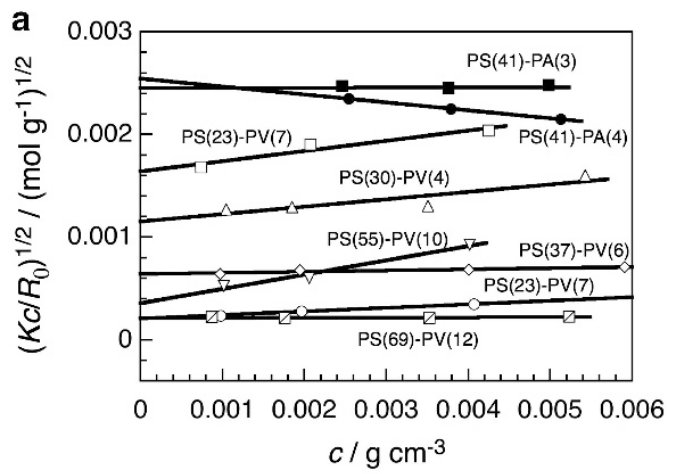

b

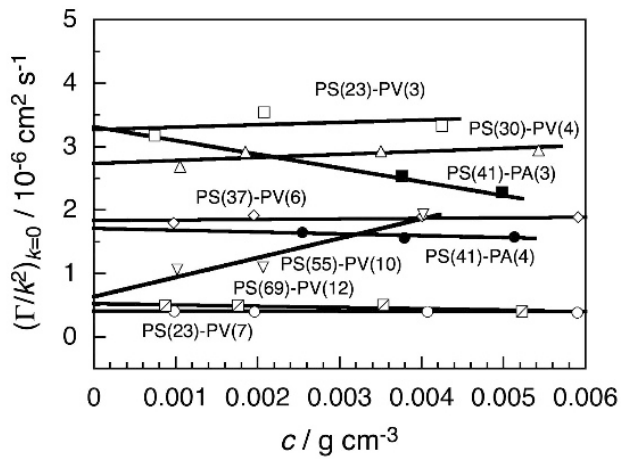

Figure 3 Concentration dependences of $\left(K c / R_{0}\right)^{1 / 2}$ (a) and $\left(\Gamma / k^{2}\right)_{k=0}$ for $\operatorname{PS}(x)-\operatorname{PV}(y)$ and PS $(x)-\operatorname{PA}(y)$ in DCE (b).

the zero $\left.k^{2}\right)$ and $\left(\Gamma / k^{2}\right)_{k=0}$ estimated from $A(\tau)$ at different $\theta$ for all of the $\operatorname{PS}(x)-\operatorname{PV}(y)$ and $\operatorname{PS}(x)-\operatorname{PA}(y)$ samples dispersible in DCE. From the straight lines indicated for the $\operatorname{PS}(x)-\operatorname{PV}(y)$ samples, the weight average molar masses $M_{\mathrm{w}}$, second virial coefficients $A_{2}$ and hydrodynamic radii $R_{\mathrm{H}}$ were determined using equations $1 \mathrm{a}$ and $\mathrm{c}$.
Table 3 Characteristics of aggregates of the PS(x)-PV(y) samples in DCE

\begin{tabular}{lccccc} 
Polymer code & $M_{w}$ & $m^{\mathrm{a}}$ & $A_{2}\left(\mathrm{~mol} \mathrm{~cm}^{3} \mathrm{~g}^{-1}\right)$ & $\left\langle S_{2}\right\rangle_{z}{ }_{z}^{1 / 2}(\mathrm{~nm})$ & $R_{H}(\mathrm{~nm})$ \\
\hline PS(23)-PV(7) & $2.4 \times 10^{7}$ & $6.3 \times 10^{3}$ & $7.26 \times 10^{-6}$ & 76 & 68 \\
PS(55)-PV(10) & $1.0 \times 10^{7}$ & $2.3 \times 10^{3}$ & $4.92 \times 10^{-5}$ & 44 & 44 \\
PS(69)-PV(12) & $2.1 \times 10^{7}$ & $2.3 \times 10^{3}$ & $2.39 \times 10^{-7}$ & 71 & 58 \\
PS(37)-PV(6) & $2.5 \times 10^{6}$ & $4.3 \times 10^{2}$ & $7.41 \times 10^{-6}$ & $-\mathrm{b}$ & 15 \\
PS(30)-PV(4) & $7.5 \times 10^{6}$ & $1.5 \times 10^{2}$ & $8.28 \times 10^{-5}$ & $-{ }^{\mathrm{b}}$ & 10 \\
PS(23)-PV(3) & $4.4 \times 10^{6}$ & $1.4 \times 10^{2}$ & $1.64 \times 10^{-4}$ & $-{ }^{\mathrm{b}}$ & 7.0 \\
\hline
\end{tabular}

Abbreviations: $m$, aggregation number; $M_{n}$, number average molecular weight; $M_{w}$ molecular weight; $R_{H}$, hydrodynamic radius; $\left\langle S_{2}\right\rangle_{Z}$, z-average square radius of gyration.

aWeight-average aggregation number $\left(M_{w} / M_{w}\right)$.

bNot determined because of very weak angular dependency.

For the PS $(x)-\operatorname{PV}(y)$ samples with $x / y=3.3,5.5$ and 5.8, the z-average radii of gyration $\left\langle S^{2}\right\rangle_{\mathrm{z}}^{1 / 2}$ were also determined from the angular dependence of $\left(K c / R_{\theta}\right)^{1 / 2}$ at $c=0$ (cf. equation $1 \mathrm{~b}$ ). In Figure 3, the concentration dependences of $\left(K c / R_{0}\right)^{1 / 2}$ and $\left(\Gamma / k^{2}\right)_{k=0}$ for two $\operatorname{PS}(x)-\mathrm{PA}(y)$ samples exhibit negative slopes, indicating that the $\operatorname{PS}(x)-\operatorname{PA}(y)$ aggregates dissociate with dilution, and the concentrations at which the light scattering measurements were performed were close to the critical micelle concentrations $(\mathrm{cmc})^{37}$ of the samples. Although PA is more solvophobic than PV, the end group in the PA block may contribute to the cmc of $\operatorname{PS}(x)-\operatorname{PA}(y)$. Because it is difficult to estimate the $M_{\mathrm{w}}$ and $R_{\mathrm{H}}$ of reverse micelle at concentrations near the $\mathrm{cmc}$ by light scattering, we did not estimate these values for $\operatorname{PS}(x)$ $\mathrm{PA}(y)$. Table 3 lists the results from the SLS and DLS measurements for the PS $(x)-\operatorname{PV}(y)$ samples in DCE. This table also contains the aggregation number $m$ ( $=M_{\mathrm{W}}$ divided by the molecular weight of each sample).

In Figure $4, R_{\mathrm{H}}$ is plotted as a function of the number $N_{0, \mathrm{w}}$ of monomer units consisting of a single aggregate for $\operatorname{PS}(x)-\operatorname{PV}(y)$ in DCE, which was calculated from $M_{\mathrm{w}}$ divided by the average monomer-unit molar mass. The data points for $\operatorname{PS}(x)-\operatorname{PV}(y)$ deviate downward from the solid line, which is indicative of the dependence of the $R_{\mathrm{H}}$ for PS on the degree of polymerization in the same solvent. (The solid line was drawn from $\left\langle S^{2}\right\rangle_{\mathrm{z}}^{1 / 2}$ data $^{38}$ with the assumed ratio $\left\langle S^{2}\right\rangle_{\mathrm{z}}^{1 / 2} / R_{\mathrm{H}}=0.775$.) This deviation may be due to the shrinkage of the PV chain and/or the branched architecture of the aggregate.

The structures of the reverse micelles formed from $\operatorname{PS}(x)-\operatorname{PV}(y)$ are discussed in detail below based on the light scattering data. If the PV block chains form a spherical core in the micelle as illustrated in Figure $5 \mathrm{a}$, the radius of the core $R_{\text {core }}$ can be calculated by

$$
\frac{4 \pi}{3} R_{\text {core }}^{3}=\frac{m}{N_{\mathrm{A}}}\left(\bar{v}_{\mathrm{v}} y M_{0, \mathrm{~V}}+\bar{v}_{\text {end }} M_{\mathrm{T}}\right)
$$

where $\bar{v}_{\mathrm{V}}$ and $\bar{v}_{\text {end }}$ are the partial specific volumes of PV and the $\operatorname{PS}(x)-\mathrm{PV}(y)$ chain ends, respectively; $M_{0, \mathrm{~V}}$ and $M_{\mathrm{T}}$ are the molar masses of the PV monomer unit and the terminal portion of the $\operatorname{PS}(x)-\operatorname{PV}(y)$ chain, respectively; and $N_{\mathrm{A}}$ is the Avogadro constant. The $\bar{v}_{\mathrm{v}}$ and $\bar{v}_{\text {end }}$ values are given in the Supplementary Information. In addition, the radius of gyration $\left\langle S^{2}\right\rangle_{\text {star }}^{1 / 2}$ of the star-like micelle (Figure 5a) can be calculated using the equation proposed by Sato and Matsuda ${ }^{39}$ from $x, m$ and $R_{\text {core }}$ (Unfortunately, there is no corresponding equation for the $R_{\mathrm{H}}$ of star micelles with a finite core size and many coronal chains.) Figure 6 compares the experimental $R_{\mathrm{H}}$ for the reverse micelles of $\operatorname{PS}(x)-\mathrm{PV}(y)$ formed in DCE with the theoretical $\left\langle S^{2}\right\rangle_{\text {star }}^{1 / 2}$. The $R_{\mathrm{H}} /\left\langle S^{2}\right\rangle_{\text {star }}{ }^{1 / 2}$ ratio is close to the $R_{\mathrm{H}} /$ $\left\langle S^{2}\right\rangle^{1 / 2}$ ratio for the uniform density sphere $(\approx 1.3)$ at $x / y>6$ but 


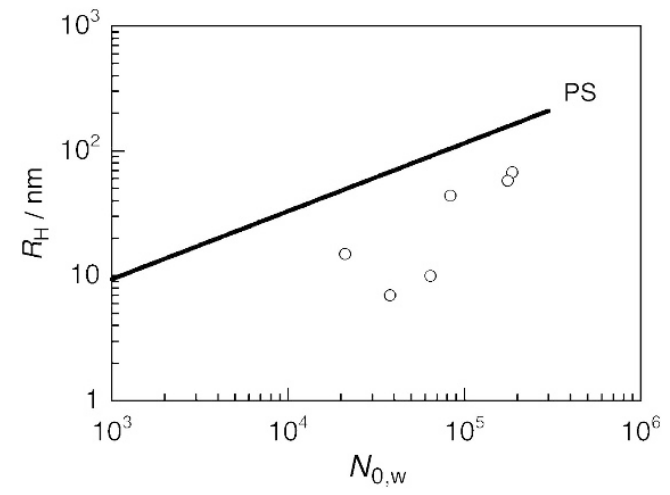

Figure $4 N_{0, w}$ dependence of $R_{\mathrm{H}}$ for $\mathrm{PS}(x)-\mathrm{PV}(y)$ in DCE (circles) compared with that for PS in the same solvent (solid line).

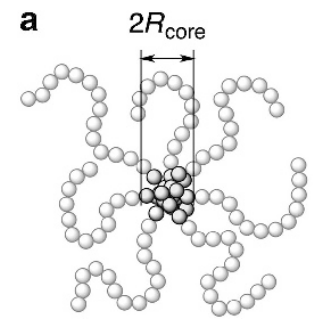

b

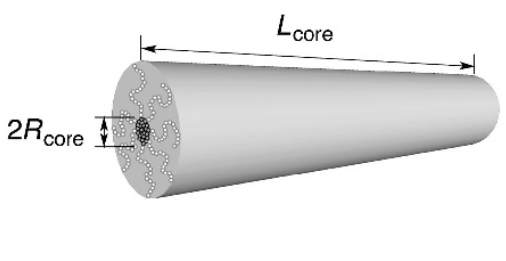

Figure 5 Conceptual illustration of star-like (a) and brush-like reverse micelles (b).

much larger than that at $x / y<6$. Figure 6 also compares the experimental $\left\langle S^{2}\right\rangle^{1 / 2}$ for reverse micelles of nonionic $\operatorname{PS}(x)$ $\operatorname{P4VP}(y)$ in toluene and $\mathrm{THF}^{30}$ as well as those for normal phase micelles of PS- $b$-poly $(N$-isopropylacrylamide) (that is, $\operatorname{PS}(y)-\mathrm{PN}(x)$ ) in water ${ }^{40}$ with the theoretical $\left\langle S^{2}\right\rangle_{\text {star }^{1 / 2}}$. Except for certain data points, the experimental $\left\langle S^{2}\right\rangle^{1 / 2}$ for $\operatorname{PS}(x)-\operatorname{P} 4 \mathrm{VP}(y)$ and $\operatorname{PS}(y)-\operatorname{PN}(x)$ were closer to the theoretical results than the experimental $R_{\mathrm{H}}$ for $\operatorname{PS}(x)-\operatorname{PV}(y)$ at smaller $x / y$.

For star-like micelles, the core surface area $a$ per copolymer chain and the distance $d$ between the nearest-neighbor coronal chains at the core-corona interface can be calculated from $R_{\text {core }}$ given by equation 2 and the experimental value $m$ by

$$
m a=4 \pi R_{\text {core }}^{2}, \quad d \equiv\left(\frac{4}{\pi} a\right)^{1 / 2}=\frac{4 R_{\text {core }}}{\sqrt{m}}
$$

Figure 7 shows the $d$ values for micelles of PS(x)-PV $(y)$ in DCE (filled circles) and $\operatorname{PS}(x)-\operatorname{P} 4 \operatorname{VP}(y)$ in toluene (squares), which are expected to be star-like micelles, with $R_{\mathrm{H}} /\left\langle S^{2}\right\rangle_{\mathrm{star}}^{1 / 2}$ (or $\left\langle S^{2}\right\rangle^{1 / 2} /\left\langle S^{2}\right\rangle_{\mathrm{star}^{1 / 2}}{ }^{1 / 2}$ ) $<2$ in Figure 6. For the $\operatorname{PS}(x)-\operatorname{P} 4 \operatorname{VP}(y)$ micelles in toluene, $d$ increased slightly with $x$. In comparison with $d$ for the $\operatorname{PS}(x)$ $\operatorname{P} 4 \operatorname{VP}(y)$ micelles, those for the $\operatorname{PS}(x)-\operatorname{PV}(y)$ micelles in DCE were considerably smaller. The diameter $d$ of the PS chain in toluene was estimated from osmotic compressibility to be $0.56 \mathrm{~nm} .{ }^{41}$ The $d$ values for the $\operatorname{PS}(x)-\operatorname{PV}(y)$ micelles were larger than but close to this thickness. Therefore, the PS coronal chains were very crowded on the core of the $\operatorname{PS}(x)-\operatorname{PV}(y)$ micelles, which is in an entropically unfavorable state but reduces the contact area between the solvophobic core and the solvent.

The formation of brush-like micelles may increase $d$ and thus the conformational entropy of PS coronal chains. If the $\operatorname{PS}(x)-\operatorname{PV}(y)$ copolymer forms a brush-like micelle as illustrated in Figure $5 \mathrm{~b}, d$ can

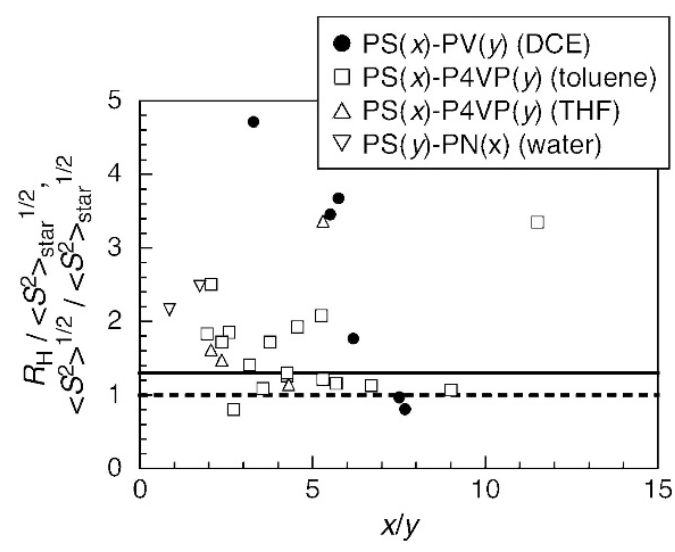

Figure $6 R_{H} /\left\langle S^{2}\right\rangle_{\mathrm{star}^{1 / 2}}{ }^{1 / 2}$ as a function of $x / y$ for PS(x)-PV(y) (filled circles) in DCE as well as $\left\langle S^{2}\right\rangle^{1 / 2} /\left\langle S^{2}\right\rangle_{\text {star }}{ }^{1 / 2}$ for PS $(x)$-P4VP(y) in toluene and THF and for $\operatorname{PS}(y)-\mathrm{PN}(x)$ in water.

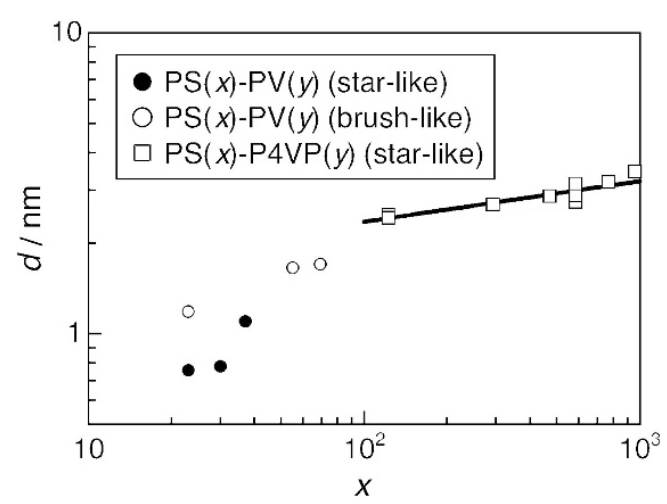

Figure $7 x$ dependence of $d$ for star-like (filled circles) and brush-like micelles (unfilled circles) formed by $\operatorname{PS}(x)-\operatorname{PV}(y)$ in DCE and star-like micelles for $\operatorname{PS}(x)-\operatorname{P4VP}(y)$ in toluene (squares).

be calculated by

$$
d=\left(\frac{8 R_{\text {core }} L_{\text {core }}}{m}\right)^{1 / 2}
$$

where the cylinder core length $L_{\text {core }}$ is calculated from the experimental radius of gyration $\left\langle S^{2}\right\rangle^{1 / 2}$ of the micelle by $L_{\text {core }}=\sqrt{12}$ $\left\langle S^{2}\right\rangle^{1 / 2}$. The unfilled circles in Figure 7 represent the $d$ values calculated using equations 2 and 4 for micelles formed from three $\operatorname{PS}(x)-\mathrm{PV}(y)$ samples, which may not be star-like micelles because $\left\langle S^{2}\right\rangle^{1 / 2}$ was much larger than $\left\langle S^{2}\right\rangle_{\text {star }}{ }^{1 / 2}$. The $d$ values expected for the brush-like micelles are considerably larger than $0.56 \mathrm{~nm}$ (the diameter of the PS chain) and comparable to the $d$ values obtained for the star-like micelles of $\operatorname{PS}(x)-\operatorname{P} 4 \operatorname{VP}(y)$. Therefore, the brush-like micelle is a possible candidate for the morphology of the micelles of $\operatorname{PS}(x)-\operatorname{PV}(y)$ where $\left\langle S^{2}\right\rangle^{1 / 2}$ is much larger than $\left\langle S^{2}\right\rangle_{\text {star }}{ }^{1 / 2}$.

\section{CONCLUSION}

In this study, amphiphilic block copolymers, $\operatorname{PS}(x)-\mathrm{PV}(y)$ with $x / y=3.3-7.7$ and $\operatorname{PS}(x)-\operatorname{PA}(y)$ with $x / y=1.2-13.7$, were prepared by RAFT radical polymerization. The block copolymer samples of $\operatorname{PS}(x)-\mathrm{PV}(y)$ exhibited a higher dispersibility in halogenated solvents than did the $\operatorname{PS}(x)-\operatorname{PA}(y)$ samples because of the higher solvophobicity of the PA block. In $\mathrm{CDCl}_{3}$, the ${ }^{1} \mathrm{H}$ NMR signals corresponded to the PV or PA blocks in the diblock copolymers, which indicated that the PS $(x)$ - 
$\operatorname{PV}(y)$ and $\operatorname{PS}(x)-\operatorname{PA}(y)$ samples formed reverse micelles in halogenated solvents. The light scattering results supported the formation of star-like reverse micelles for $\operatorname{PS}(x)-\mathrm{PV}(y)$ with $x / y>6$ in DCE and brush-like reverse micelles for $\operatorname{PS}(x)-\operatorname{PV}(y)$ with $x / y<6$ in the same solvent.

\section{ACKNOWLEDGEMENTS}

This work was partially supported by Grant-in-Aid for Scientific Research No. 23350055 and 23550137 from the Japan Society for the Promotion of Science.

1 Hashidzume, A., Morishima, Y. \& Szczubiałka, K. in Handbook of Polyelectrolytes and Their Applications (eds Tripathy, S. K., Kumar, J. \& Nalwa, H. S.) Vol. 2, Ch. 1 1-63 (American Scientific Publishers, Stevenson Ranch, CA, USA, 2002).

2 McCormick, C. L., Bock, J. \& Schulz, D. N. in Encyclopedia of Polymer Science and Engineering (ed. Kroschwitz, J. I.) Vol. 17, 730-784 (Wiley \& Sons, New York, NY USA, 1989).

3 Laschewsky, A. Molecular concepts, self-organisation and properties of polysoaps. Adv Polym. Sci. 124, 1-86 (1995).

4 Okhapkin, I. M., Makhaeva, E. E. \& Khokhlov, A. R. Water solutions of amphiphilic polymers: Nanostructure formation and possibilities for catalysis. Adv. Polym. Sci. 195, 177-210 (2006).

5 Glass, J. E. Hydrophilic polymers. Performance with Environmental Acceptability Advances in Chemistry Series 248 (American Chemical Society, Washington, DC, USA 1996).

6 Glass, J. E. Associative Polymers in Aqueous Solutions ACS Symposium Series 765American Chemical Society, Washington, DC, USA, 2000).

7 McCormick, C. L. Stimuli-responsive Water Soluble and Amphiphilic Polymers ACS Symposium Series 780 (American Chemical Society, Washington, DC, 2001).

8 Tuzar, Z. \& Kratochvíl, P. Block and graft copolymer micelles in solution. Adv. Colloid Interface Sci. 6, 201-232 (1976).

9 Qin, A., Tian, M., Ramireddy, C., Webber, S. E., Munk, P. \& Tuzar, Z. Polystyrenepoly(methacrylic acid) block copolymer micelles. Macromolecules 27, 120-126 (1994).

$10 \mathrm{Chu}, \mathrm{B}$. Structure and dynamics of block copolymer colloids. Langmuir. 11, 414-421 (1995).

11 Förster, S., Zisenis, M., Wenz, E. \& Antonietti, M. Micellization of strongly segregated block copolymers. J. Chem. Phys. 104, 9956-9970 (1996)

12 Moffitt, M., Khougaz, K. \& Eisenberg, A. Micellization of ionic block copolymers. Acc. Chem. Res. 29, 95-102 (1996)

13 Förster, S. \& Antonietti, M. Amphiphilic block copolymers in structure-controlled nanomaterial hybrids. Adv. Mater. (Weinheim, Ger.) 10, 195-217 (1998).

14 Förster, S., Abetz, V. \& Müller, A. H. E. Polyelectrolyte block copolymer micelles. Adv. Polym. Sci. 166, 173-210 (2004).

15 Chen, D. \& Jiang, M. Strategies for constructing polymeric micelles and hollow spheres in solution via specific intermolecular interactions. Acc. Chem. Res. 38, 494-502 (2005).

16 Choucair, A., Lavigueur, C. \& Eisenberg, A. Polystyrene-b-poly(acrylic acid) vesicle size control using solution properties and hydrophilic block length. Langmuir. 20, 3894-3900 (2004)

17 Aoshima, S., Sugihara, S., Shibayama, M. \& Kanaoka, S. Synthesis and selfassociation of stimuli-responsive diblock copolymers by living cationic polymerization. Macromol. Symp. 215, 151-163 (2004).

18 Mitsukami, Y., Hashidzume, A., Yusa, S.-i., Morishima, Y., Lowe, A. B. \& McCormick, C. L. Characterization of $\mathrm{pH}$-dependent micellization of polystyrene-based cationic block copolymers prepared by reversible addition-fragmentation chain transfer (RAFT) radical polymerization. Polymer. (Guildf). 47, 4333-4340 (2006).

19 Morishima, Y. Thermally responsive polymer vesicles. Angew. Chem. Int. Ed. 46, 1370-1372 (2007).
20 Chung, B., Choi, M., Ree, M., Jung, J. C., Zin, W. C. \& Chang, T. Subphase pH effect on surface micelle of polystyrene- $b$-poly(2-vinylpyridine) diblock copolymers at the airwater interface. Macromolecules 39, 684-689 (2006).

21 Smith, A. E., Xu, X. \& McCormick, C. L. Stimuli-responsive amphiphilic (co)polymers via RAFT polymerization. Prog. Polym. Sci. 35, 45-93 (2010).

22 Cheng, F., Yang, X., Peng, H., Chen, D. \& Jiang, M. Well-controlled formation of polymeric micelles with a nanosized aqueous core and their applications as nanoreactors. Macromolecules 40, 8007-8014 (2007).

23 Zhang, L. \& Eisenberg, A. Crew-cut aggregates from self-assembly of blends of polystyrene- $b$-poly(acrylic acid) block copolymers and homopolystyrene in solution. J. Polym. Sci., Part B: Polym. Phys. 37, 1469-1484 (1999).

24 Spatz, J. P., Mößmer, S. \& Möller, M. Metastable reverse globular micelles and giant micellar wires from block copolymers. Angew. Chem. Int. Ed. Engl. 35, 1510-1512 (1996).

25 Nguyen, D., Williams, C. E. \& Eisenberg, A. Block ionomer micelles in solution. 1. Characterization of ionic cores by small-angle X-ray scattering. Macromolecules 27, 5090-5093 (1994).

26 Zhang, L. \& Eisenberg, A. Multiple morphologies of "crew-cut" aggregates of polystyrene-b-poly(acrylic acid) block copolymers. Science 268, 1728-1731 (1995).

27 Zhong, X. F., Varshney, S. K. \& Eisenberg, A. Critical micelle lengths for ionic blocks in solutions of polystyrene- $b$-poly(sodium acrylate) ionomers. Macromolecules 25, $7160-7167$ (1992)

28 Desjardins, A. \& Eisenberg, A. Colloidal properties of block ionomers. 1. Characterization of reverse micelles of styrene- $b$-metal methacrylate diblocks by size-exclusion chromatography. Macromolecules 24, 5779-5790 (1991).

29 Desjardins, A., van de Ven, T. G. M. \& Eisenberg, A. Colloidal properties of block ionomers. 2. Characterization of reverse micelles of styrene- $b$-methacrylic acid and styrene- $b$-metal methacrylate diblocks by dynamic light scattering. Macromolecules 25, 2412-2421 (1992).

30 Antonietti, M., Heinz, S., Schmidt, M. \& Rosenauer, C. Determination of the micelle architecture of polystyrene/poly(4-vinylpyridine) block copolymers in dilute solution. Macromolecules 27, 3276-3281 (1994).

31 Thang, S. H., Chong, Y. K., Mayadunne, R. T. A., Moad, G. \& Rizzardo, E. A nove synthesis of functional dithioesters, dithiocarbamates, xanthates and trithiocarbonates. Tetrahedron Lett. 40, 2435-2438 (1999).

32 Hashidzume, A., Kawaguchi, A., Tagawa, A., Hyoda, K. \& Sato, T. Synthesis and structural analysis of self-associating amphiphilic statistical copolymers in aqueous media. Macromolecules 39, 1135-1143 (2006).

33 Kanao, M., Matsuda, Y. \& Sato, T. Characterization of polymer solutions containing a small amount of aggregates by static and dynamic light scattering. Macromolecules 36, 2093-2102 (2003).

34 Kawata, T., Hashidzume, A. \& Sato, T. Micellar structure of amphiphilic statistical copolymers bearing dodecyl hydrophobes in aqueous media. Macromolecules 40 , 1174-1180 (2007)

35 Utiyama, H., Takenaka, K. Mizumori, M. \& Fukuda, M. Light-scattering studies of a polystyrene-poly(methyl methacrylate) two-block copolymer in dilute solutions. Macromolecules 7, 28-34 (1974).

36 Tanaka, T., Kotaka, T. \& Inagaki, H. Intermolecular correlation in light scattering from dilute solutions of block copolymers. Macromolecules 7, 311-319 (1974).

37 Israelachvili, J. N. In Intermolecular and surface forces. 2nd ed. Ch. 16 (Academic Press, London, 1992)

38 Outer, P., Carr, C. I. \& Zimm, B. H. Light scattering investigation of the structure of polystyrene. J. Chem. Phys. 18, 830-839 (1950).

39 Sato, T. \& Matsuda, Y. Macromolecular assemblies in solution: Characterization by light scattering. Polym. J. (Tokyo, Jpn.) 41, 241-251 (2009).

40 Zhang, W., Zhou, X., Li, H., Fang, Y. \& Zhang, G. Conformational transition of tethered poly $(\mathrm{N}$-isopropylacrylamide) chains in coronas of micelles and vesicles. Macromolecules 38, 909-914 (2005).

41 Koyama, R. \& Sato, T. Thermodynamic properties of toluene solutions of low molecular weight polystyrenes over wide ranges of concentration. Macromolecules 35 2235-2242 (2002)

Supplementary Information accompanies the paper on Polymer Journal website (http://www.nature.com/pj) 